\title{
Diagnóstico de las Dificultades de la Expresión Oral de los Estudiantes de Nivel B1 de la Alianza Francesa de San Luis Potosí/México
}

\section{Diagnosis of the Difficulties of the Oral Expression of the B1 Level Students of the French Alliance of San Luis Potosí / Mexico}

\author{
Ismaila Mounkoro $^{1 \mathrm{a}}$ y Sergio Raúl Herrera $\mathrm{Meza}^{2}$ \\ Centro Universitario CIFE, México ${ }^{12}$
}

(D) ORCID ID: https://orcid.org/0000-0003-4198-9583 ${ }^{1}$

(iD) ORCID ID: https://orcid.org/0000-0003-2605-4878²

Recibido: 01 de setiembre de 2020

Aceptado: 23 de diciembre de 2020

\begin{abstract}
Resumen
El propósito de este artículo fue diagnosticar las dificultades en la competencia oral que presentan los estudiantes de francés como lengua extranjera aspirantes al nivel B1 de Alianza Francesa de San Luis Potosí (México). Se realizó un estudio cuantitativo y cualitativo, se emplearon dos instrumentos para la recolecta de los datos: una rúbrica analítica socioformativa para evaluar la competencia oral a través de cinco componentes (la pronunciación, el vocabulario, la coherencia y cohesión, la competencia gramatical y la interacción.) y se realizaron unas entrevistas semiestructuradas a 17 evaluadores. Participaron en la evaluación 247 estudiantes hispanoparlantes de entre 13 y 45 años. Los resultados obtenidos demostraron que la principal dificultad es la falta de vocabulario y los factores encontrados son la influencia de la lengua materna y de una segunda lengua que es el inglés, así como el temor a los maestros nativos. Se sugieren estudios a profundidad sobre cada una de las dificultades encontradas.
\end{abstract}

Palabras clave: Alianza Francesa, competencia oral, diagnóstico, dificultades, evaluación socioformativa

\begin{abstract}
${ }^{\mathrm{a}}$ Correspondencia al autor:

E-mail: mounkorosoumi@gmail.com
\end{abstract}

The purpose of this article was to diagnose the difficulties in oral competence presented by students of French as a foreign language aspiring to level B1 of Alliance Française in San Luis Potosí (Mexico). A quantitative and qualitative study was carried out, two instruments were used to collect the data: a socioformative analitic rubric to evaluate oral competence through five components (pronunciation, vocabulary, coherence and cohesion, grammatical competence and interaction) and semi-structured interviews were conducted with 17 evaluators. A sample of 247 Spanish-speaking students aged between 13 and 45 participated in the evaluation. The 
results showed that the main difficulty is the lack of vocabulary and the factors found are the influence of the mother tongue and a second language that is English, as well as fear of native teachers. In-depth studies are suggested on each of the difficulties encountered.

Keywords: Diagnostics, difficulties, Alliance Française, oral competence, socioformative evaluation,

\section{Introducción}

En 1883, se crea la Alianza Francesa de París (Francia) con el objetivo de difundir la cultura francesa en el extranjero a través de la enseñanza de la lengua francesa. La Alianza Francesa de México fue una de las primeras en abrir fuera de Francia, hace más de 130 años. A través del tiempo la red de la Alianza Francesa en México fue extendiéndose hasta llegar a contar, hoy en día, con 33 sedes que enseñan el idioma francés a más de 25 mil alumnos de todo el mundo cada año. En la ciudad de San Luis Potosí, México, fue fundada en 1948 y actualmente cuenta con 25 profesores de diferentes nacionalidades y más de 400 estudiantes.

Con el propósito de mejorar la enseñanza, garantizar la calidad de los servicios ofrecidos y transformar el sistema de enseñanza hacia la sociedad del conocimiento, se lleva a cabo, regularmente, la evaluación del desempeño de los estudiantes a fin de detectar los aspectos que necesitan mejorarse de parte de los docentes y de la institución.

Existen distintas formas de referirse al concepto de hablar en lengua extranjera, Bygates (1991) usa la expresión comunicación oral y la define como la habilidad de unir frases en lo abstracto para producirlas y adaptarlas en las diversas circunstancias, es decir, poder tomar decisiones rápidas, adecuadas y ajustadas en función de la situación. O’Maley y Valdez (1996) usan la expresión producción oral con la que se refieren a la habilidad de negociar significados entre dos o más personas que están relacionadas al contexto donde ocurre la conversación. Por su parte, Ramírez (2002) define la habilidad oral como una competencia que forma parte de la interacción oral. Según él, la interacción es una actividad de comunicación en la que dos o más personas participan y tienen una influencia mutua en el intercambio.

El Marco Común Europeo (MCER) utiliza la expresión habilidad de hablar y la define como la producción de un texto oral recibido por una o más personas. En el MCER la habilidad de hablar está dividida en expresión oral e interacción oral. Precisa que las dos expresiones se utilizan en situaciones diferentes. El término expresión oral se utiliza cuando se trata de actividades, tales como comunicados públicos, sermones, espectáculos, comentarios 
deportivos, presentaciones de ventas, descripción de experiencias, entre otros y la interacción oral cuando se trata de actividades como colaborar para alcanzar un objetivo, interactuar para obtener bienes y servicios, intercambiar información, entrevistar y ser entrevistado, entre otros (Consejo Europeo, 2002).

En este estudio se considera la competencia oral, ya que es un concepto más completo que abarca todos los componentes lingüísticos y no lingüísticos. Según Níkleva \& López-García (2019) la competencia oral es la habilidad lingüística relacionada con la producción del discurso oral e abarca el dominio de la pronunciación, del léxico, la gramática de la lengua meta y los conocimientos socioculturales y pragmáticos.

Varios autores mencionan que la competencia oral es la parte que requiere la mayor atención cuando se aprende una segunda lengua (Oxford, 1990; Scrivener, 2005; Ur, 1996; Níkleva \& López-García, 2019) porque hablar es el principal medio a través del cual los estudiantes enfrentan de forma cotidiana los retos de la sociedad del conocimiento.

Ahora, se han hecho varias investigaciones sobre las dificultades de la competencia oral, por ejemplo, Al Hosni (2016) investigo sobre los problemas y dificultades de la competencia oral que encuentran los estudiantes de idiomas en la Universidad Abierta Al Quds. Eligió una muestra aleatoria de la población de estudio compuesta por (14) estudiantes que estudian Idioma en Inglés.

Asma (2016) presenta una tesis de maestría sobre las dificultades de la competencia oral de los estudiantes de la universidad de Mohamed Khider en Algeria. Consistió en un test oral aplicado a 20 estudiantes que llevaban entre 400 y 500 horas de estudio del francés como segunda lengua. Hrisa y Pop-Pacurar (2016) trabajaron sobre los motivos que causan una barrera para la comunicación efectiva entre estudiantes y profesores en lengua extranjera. Al igual que otros estudios, se aplicó un cuestionario de opción múltiple a 169 estudiantes de pregrado y posgrado de la universidad Babes-Bolyai en Rumania para conocer sus opiniones sobre el problema.

Yalçın y Volkan (2014) realizaron una investigación para identificar los factores que causan dificultades en la competencia oral por medio de juegos, dramatizaciones y debates. Al final aplicaron un cuestionario a los 12 participantes de entre 18 y 24 años de edad, todos estudiantes de inglés del departamento de enseñanza de idiomas en una universidad privada en Estambul, para que señalaran en qué parte tuvieron dificultades y así detectar los factores más inciden en tales dificultades. Al Mustafá (2016) investigó en su trabajo de tesis de maestría las 
dificultades que en la competencia oral presentan los estudiantes de la carrera de Ciencia y Tecnología en la universidad de Soudan. Realizó un test oral con 20 estudiantes que llevan entre 400 y 500 horas de estudio del francés, nivel B1 según el Marco Común Europeo de las lenguas.

Sánchez (2016), en su trabajo de tesis sobre los obstáculos en el aprendizaje del inglés como lengua extranjera, realizo un estudio descriptivo con una muestra de 60 estudiantes con edades entre 12 y 18 años y 60 adultos con un rango de edad entre 45 y 60 años y encontró una falta de entrenamiento de los estudiantes a manejar las emociones negativas durante la producción oral y concluyo que los factores emocionales son tan determinantes en este proceso como los cognitivos.

Níkleva; \& López-García (2019), realizaron un trabajo de reflexión crítica sobre las dificultades que se presentan para la enseñanza de la expresión oral en el aula de Educación Primaria. Observaron una serie de fundamentos teóricos para el desarrollo de la expresión oral y encontraron tres principales dificultades que son: El plano del mensaje (unidad temática, generalizaciones y cuestiones propias del discurso oral; el plano del lenguaje (niveles linguiísticos: fonológico, morfológico, sintáctico, léxico-semántico; y el plano de los mecanismos psicológicos básicos: la personalidad del hablante (tímidos, verborrágicos, interruptores, etc.)

Mejía (2014), estudió de manera exploratoria, la incidencia que tienen los factores de motivación, ansiedad, estilos y estrategias de aprendizaje en el lento y difícil aprendizaje del inglés que se observa en algunos estudiantes de la Universidad Icesi. Trabajó con diez estudiantes de la Universidad Icesi que presentan dificultades en el aprendizaje del inglés como lengua extranjera, indagando cómo se percibían en estas áreas a través de grupos focales y entrevistas en profundidad. Llego a la conclusión que los estudiantes que presentan dificultades manejan niveles altos de ansiedad, usan estrategias metacognitivas y sociales en menor medida, hacen una evaluación negativa del docente y/o del curso de inglés y tienen niveles más bajos de intensidad motivacional que los estudiantes con buen desempeño.

Youcef, (2018) trabajo sobre los origines de las dificultades en la producción oral de los estudiantes de maestría en la universidad Guelma en Algeria, utilizó un cuestionario destinado a estudiantes de primer año de maestría en francés y observó el grupo durante un curso de producción oral . Llego a la conclusión que la falta de dominio del idioma es la causa de las obstáculos psicológicos y eso se expresa con el miedo escénico y el miedo a hablar y la timidez. 
Molina \& Jiménez-Fernández (2015), examinaron la posible existencia de una dificultad específica para adquirir una segunda lengua, elaboraron y aplicaron una entrevista a docentes especialistas. Sus resultados mostraron que la falta de motivación, alumnos desinteresados en la asignatura y/o con baja atención en clase, poca responsabilidad y esfuerzo para realizar las tareas, problemas en el ambiente familiar, niños con desventaja sociocultural, extrema inmadurez; niños que son más infantiles que sus compañeros como fuentes de las dificultades.

Como se puede observar, todos esos estudios fueron efectuados por medio de cuestionario y en la mayoría son los estudiantes que señalan sus dificultades. Se necesita realizar procesos de evaluación diagnóstica directamente sobre el empleo de la lengua en la comunicación oral, a través de un evaluador capacitado y empleando instrumentos validados, a fin de evitar imprecisiones por la apreciación subjetiva de los sujetos evaluados y así poder detectar con rigor científico y precisión las dificultades más importantes y los factores que en ellas influyen.

Entendemos que la competencia oral es la capacidad de usar las herramientas comunicativas necesarias para llevar a cabo una tarea que involucra el empleo del lenguaje oral. Eso requiere el conocimiento de diferentes componentes tantos lingüísticos como extralingüísticos, como son: la gramática, la pronunciación, el vocabulario, la coherencia y cohesión, y la interacción. La competencia gramatical es la habilidad de usar correctamente el código lingüístico. La pronunciación es la articulación de los sonidos para producir palabras entendibles. El vocabulario es el conjunto de palabras que se usa en una lengua, permite expresar ideas claras y precisas. La coherencia y cohesión consiste en saber relacionar las diferentes partes al interior de un discurso, así como relacionar el propio discurso con el de los otros interlocutores y así producir y comprender mensajes significativos. La interacción es la capacidad de usar correctamente los diferentes significados en función de la situación de comunicación, por ejemplo, la diferencia de registro cuando nos dirigimos a un amigo o a un superior. Es decir que este componente implica el dominio de reglas socioculturales, registro y variedades lingüísticas (Consejo Europeo, 2002).

El MCER clasifica los diversos niveles de domino en el uso de la lengua en una escala de tres niveles y dos subniveles en cada uno de ellos. Así, el nivel A corresponde a un uso básico, y el nivel C al más alto. De acuerdo con esta clasificación, el nivel B1 es el que corresponde a usuarios que se comunican con seguridad en asuntos tanto habituales como poco habituales relacionados con sus intereses y su especialidad. El usuario B1 tiene un repertorio 
lingüístico sencillo para enfrentarse a la mayoría de las situaciones que pueden surgir cuando se viaja, participa sin preparación previa en conversaciones que traten temas cotidianos, expresa opiniones e intercambia información sobre temas habituales de interés personal (Consejo Europeo, 2002). Se eligió el nivel B1 porque los estudiantes de este nivel son los que manifestaron más dificultades en las encuestas de satisfacción que se aplica a los estudiantes al final de cada módulo y también porque la mayoría de ellos tienen proyectos de realizar alguna experiencia de intercambio en un país francófono y el B1 es el nivel de dominio mínimo requerido.

La socioformación es un enfoque educativo desarrollado en Latinoamérica para potencializar el proceso de enseñanza-aprendizaje con la finalidad de transformar la sociedad hacia una sociedad del conocimiento, es decir formar una sociedad con un nivel óptimo de calidad de vida a través de la realización de trabajos colaborativos en proyectos e implicando la convivencia pacífica, el desarrollo socioeconómico y la sostenibilidad ambiental (Tobón, Guzmán, Hernández, \& Cardona, 2015).

Para la socioformación, la evaluación es todo un proceso formativo y no solo una etapa final de un curso, que tiene el único propósito de otorgar una calificación (Tobón, 2013). Una de las finalidades principales de la evaluación es la de realizar un diagnóstico de los logros previos y las necesidades de apoyo (Tobón, 2014), es decir, cómo están los estudiantes en la adquisición y el dominio de los diferentes componentes de cada habilidad y cuáles son sus intereses y expectativas, con el fin de articular las estrategias didácticas y actividades pertinentes para el logro de las metas del proceso formativo.

En lo que se refiere al aprendizaje de la lengua, la evaluación diagnóstica es un instrumento de retroalimentación sobre los objetivos del curso. Se hace en general al inicio del curso y sirve como medio de orientación y de localización de problemas.

La socioformación entiende que todo proceso de evaluación tiene una finalidad formativa Cuando se trata de evaluar competencias, que son unidades de análisis complejas ya que expresan actuaciones integrales para la resolución de algún problema del contexto (Tobón, 2013; Zabalza, 2017). La socioformación propone el empleo de instrumentos tipo rúbrica, ya que son los que permiten detectar con mayor precisión distintos niveles de desempeño (Tobón, 2017). Para determinar la gradualidad en los niveles de desempeño, la socioformación propone una taxonomía de cinco niveles: preformal, receptivo, resolutivo, autónomo y estratégico, que en términos generales va desde un desempeño muy elemental y dependiente de la asistencia de 
alguna otra persona (preformal y receptivo) hasta un desempeño fluido caracterizado por la seguridad y la creatividad (autónomo y estratégico) (Tobón, 2015).

En el caso específico del domino de una lengua, los niveles de la taxonomía socioformativa pueden describirse, en términos generales, de la siguiente manera: en el nivel preformal, los alumnos enuncian un discurso muy simple y las informaciones presentadas son expresadas de forma memorística y textual; en el nivel receptivo reproducen un discurso simple, necesitando corregir y reformular sus expresiones continuamente: en el nivel resolutivo desarrollan discursos más elaborados y bien estructurados; en el nivel autónomo formulan un discurso claro y completo y suficientemente fluido; y en el nivel estratégico componen discursos claros y precisos capaces de generar interés, expresados con seguridad y pronunciados de forma aproximada al hablante nativo

Desde esta visión innovadora de la evaluación socioformativa, se llevó a cabo una investigación entre estudiantes de francés como lengua extranjera o segunda lengua con las siguientes metas:

1. Identificar con precisión las dificultades que experimentan los estudiantes de la Alianza Francesa de San Luis Potosí para el desempeño de la competencia oral.

2. Conocer los principales factores que contribuyen a la existencia de estas dificultades.

El propósito final es contar con elementos desde los que se puedan generar estrategias de transformación y mejora de los procesos formativos para el desarrollo de las habilidades comunicativas de los estudiantes de francés en contextos hispanoparlantes, específicamente las relacionadas con la competencia oral.

\section{Metodología}

\section{Tipo de Estudio}

Se realizó una investigación de tipo mixto recolectando y analizando datos cuantitativos y cualitativos de la evaluación de los participantes mediante la rúbrica socioformativa y analizando, con técnicas cualitativas el discurso de los evaluadores en entrevistas.

\section{Participantes}

Los participantes fueron los alumnos de la Alianza Francesa de San Luis Potosí del ciclo escolar 2019-2020 que asisten a los cursos que tienen el objetivo de que los estudiantes 
alcancen el nivel B1. La muestra estuvo constituida por 247 estudiantes, 168 mujeres y 79 hombres con edades de entre 13 y 45 años. En la Tabla 1 se presenta sus características. También se contó con la participación de 17 docentes, instructores de los evaluados para la aplicación del examen y, a su vez, fueron los sujetos entrevistados. La mayoría de ellos son examinadores y correctores de certificaciones oficiales en lenguas extranjeras, quienes tienen, como mínimo, el grado de licenciatura y cuentan con al menos tres años de experiencia en la docencia. En la Tabla 2 se presentan sus características. El objetivo de la entrevista fue obtener más información respecto a los factores que están relacionados con las dificultades que los estudiantes enfrentan cuando intentan hablar francés, pero desde la perspectiva de los profesores.

\section{Tabla 1}

Características sociodemográficas de los participantes

\begin{tabular}{ll}
\hline Características & Datos \\
\hline $\mathrm{N}$ & 247 \\
Sexo & $27.13 \%$ hombres \\
& $72.87 \%$ mujeres \\
Estado civil & $80.97 \%$ soltero /soltera \\
& $08.10 \%$ unión libre \\
& $10.93 \%$ casado/casada \\
Ocupación & $92.72 \%$ Estudiantes \\
& $07.28 \%$ Docentes \\
Nivel socioeconómico & $67.61 \%$ Bueno \\
Lenguas Extranjeras que hablan & $32.39 \%$ Medio \\
& $19.02 \%$ Una \\
& $67.62 \%$ Dos \\
& $13.36 \%$ Tres \\
\hline
\end{tabular}

Elaboración propia 


\section{Tabla 2}

Características de los evaluadores

\begin{tabular}{|c|c|c|c|c|c|}
\hline Evaluadores & Sexo & Grado & Cargo & $\begin{array}{c}\text { Años experiencias } \\
\text { Como docente }\end{array}$ & $\begin{array}{l}\text { Evaluador } \\
\text { certificado }\end{array}$ \\
\hline Evaluador 1 & Hombre & Maestría & $\begin{array}{c}\text { Guía } \\
\text { pedagógico }\end{array}$ & 34 & Sí \\
\hline Evaluador 2 & Hombre & Maestría & Docente & 13 & Sí \\
\hline Evaluador 3 & Mujer & Maestría & Docente & 8 & Sí \\
\hline Evaluador 4 & Hombre & Licenciatura & Docente & 3 & Sí \\
\hline Evaluador 5 & Hombre & Licenciatura & Docente & 4 & Sí \\
\hline Evaluador 6 & Mujer & Maestría & Directora & 28 & Sí \\
\hline Evaluador 7 & Hombre & Maestría & Coordinador & 18 & Sí \\
\hline Evaluador 8 & Hombre & Maestría & Docente & 15 & Sí \\
\hline Evaluador 9 & Hombre & Licenciatura & Docente & 3 & Sí \\
\hline Evaluador 10 & Hombre & Maestría & Docente & 7 & Sí \\
\hline Evaluador 11 & Hombre & Maestría & Docente & 2 & No \\
\hline Evaluador 12 & Hombre & Maestría & Docente & 4 & Sí \\
\hline Evaluador 13 & Mujer & Maestría & Docente & 7 & No \\
\hline Evaluador 14 & Mujer & Maestría & Docente & 22 & Sí \\
\hline Evaluador 15 & Hombre & Maestría & Docente & 13 & Sí \\
\hline Evaluador 16 & Hombre & Licenciatura & Docente & 2 & Sí \\
\hline Evaluador 17 & Hombre & Licenciatura & Docente & 3 & Sí \\
\hline
\end{tabular}




\section{Procedimiento}

Se lanzó la convocatoria para todos los profesores del nivel B1, a quienes se les hizo llegar un link de la rúbrica con fechas y horas de evaluación, ellos a su vez se la hicieron llegar a sus alumnos. El procedimiento de la evaluación fue el siguiente:

1. El examinado tomó dos tarjetas con los posibles temas a elegir.

2. Una vez examinadas las dos tarjetas, eligió el tema de su preferencia.

3. Contó con un tiempo de 10 minutos para preparar la presentación del tema;

4. Al pasar con los examinadores:

- Contó con un máximo de 2 minutos para presentar el tema

- En 3 a 5 minutos debió dar su punto de vista

- Durante otros 5 minutos sostuvo una conversación con el examinador.

Los profesores que aplicaron las evaluaciones fueron entrevistados sobre dos ejes principales: las dificultades de la competencia oral y los factores que influyen en esas dificultades.

\section{Instrumentos}

Se utilizaron dos instrumentos: una rúbrica socioformativa para evaluar la competencia oral en una segunda lengua, validada en su contenido y constructo (Mounkoro \& HerreraMeza, 2019) y una entrevista a los evaluadores. Durante la entrevista se realizó las siguientes preguntas:

1-¿Cuales son según usted las dificultades de vocabulario que encuentran los estudiantes de nivel B1 en francés? ¿Cuáles son según usted las causas de esas dificultades y que solución propone?

2-¿Cuales son según usted las dificultades gramaticales que encuentran los estudiantes de nivel B1 en francés? ¿Cuáles son según usted las causas de esas dificultades y que solución propone?

3-¿Cuales son según usted las dificultades de pronunciación que encuentran los estudiantes de nivel B1 en francés? ¿Cuáles son según usted las causas de esas dificultades y que solución propone?

4-¿Cuales son según usted las dificultades de Coherencia y cohesión que encuentran los estudiantes de nivel B1 en francés? ¿Cuáles son según usted las causas de esas dificultades y que solución propone? 
La rúbrica socioformativa considera los siguientes componentes: a) la pronunciación y fluidez, b) el vocabulario, c) la corrección gramatical, d) la coherencia y cohesión, y e) la interacción (Figura 1). Las opciones de respuesta son descriptores de cinco niveles de desempeño de la competencia oral, de acuerdo con la taxonomía socioformativa: preformal, receptivo, resolutivo, autónomo y estratégico (Tabla 3). Durante la entrevista se tomó nota mientras se hablaba con los encuestados para su posterior análisis.

El proceso de diseño del instrumento fue lo siguiente, se realizó una búsqueda de herramientas que evaluaran la competencia oral de una lengua extranjera en los bases de datos Google Scholar, Redalyc, IRESIE, SCIELO, WoS, SCOPUS, entre otros y después se procedió al diseño del instrumento considerando los siguientes referentes teóricos y metodológicos: Evaluación con rúbrica socioformativa, Las escalas analíticas de los exámenes DELE; El MCER para las lenguas. A partir de los referentes citados se diseñó el instrumento compuesto con los 5 ítems y una sola dimensión: la competencia oral. Posterior al diseño, se solicitó el apoyo de tres expertos para la mejora del instrumento. Una vez diseñado el instrumento por el equipo de investigadores, los expertos revisaron y analizaron los descriptores.

En cuanto al proceso de validación fue en 3 etapas, la primera fue el Estudio de la validez de contenido que consistió en la evaluación del instrumento por parte de 15 jueces seleccionados bajo los criterios de grado académico, una experiencia mínima de 10 años en el área de investigación, ser autor de al menos un artículo y tener experiencia en el diseño y/o validación de instrumentos de investigación. Ellos evaluaron cuantitativamente la calidad de la redacción de cada ítem mediante una escala de cuatro niveles según el grado de comprensibilidad. El análisis de la evaluación cuantitativa se realizó mediante el coeficiente de concordancia V de Aiken y estuvo un índice V de Aiken superior a 0.75 en los dos criterios que fueron evaluados: pertinencia y redacción. Después del juicio de expertos, se aplicó la prueba a un grupo piloto compuesto por 17 docentes de idiomas con más de un año de experiencia, la mayoría de ellos examinadores de certificaciones oficiales en lenguas extranjeras. Evaluaron una estudiante de nivel B1 y después respondieron a una encuesta de satisfacción del instrumento sobre redacción y claridad del instrumento. Una vez que se verificó que la rúbrica tenía buenos niveles de pertinencia, redacción y satisfacción en el grupo piloto, se procedió a realizar el análisis de validez de constructo por medio de un análisis factorial exploratorio (AFE). Por último, se determinó la confiabilidad de la aplicación de la 
rúbrica mediante el coeficiente de Alfa de Cronbach que fue de 0.842 para la prueba piloto y 0.916 en la aplicación de la prueba masiva.

Figura 1. Los componentes de la rúbrica

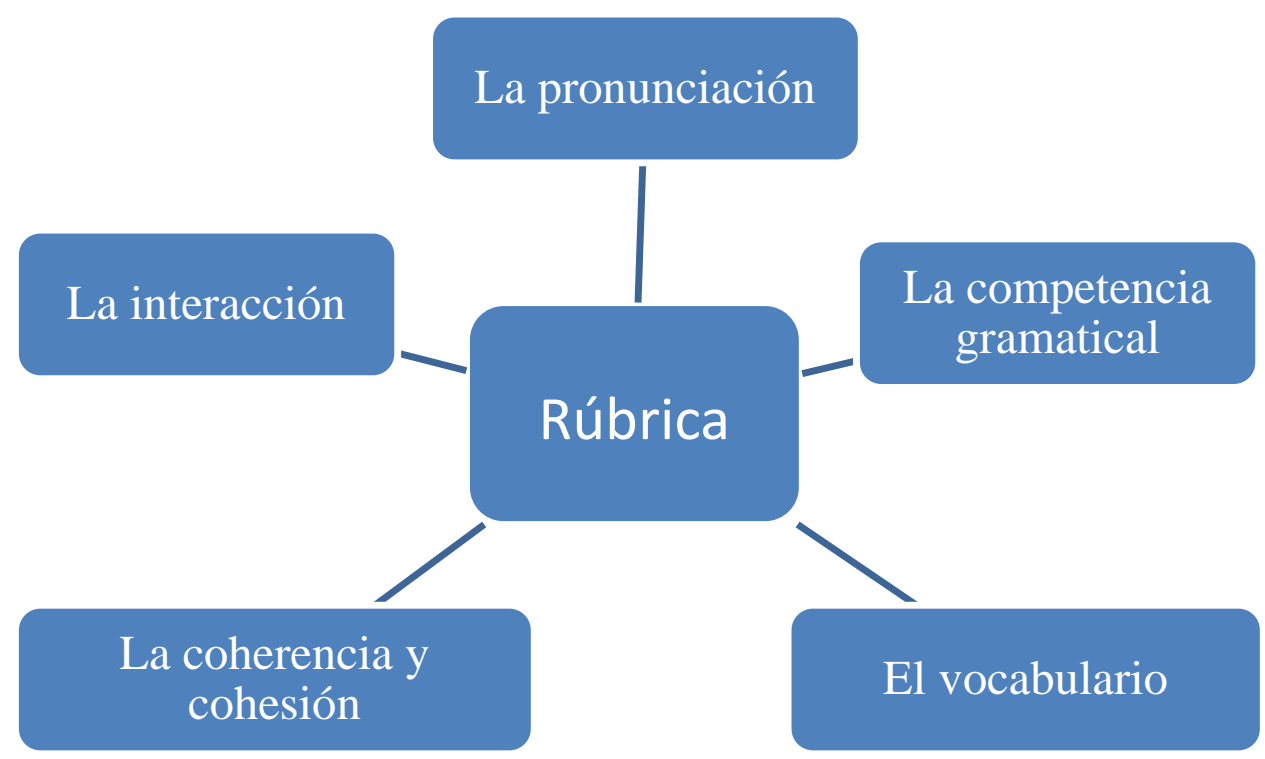

Tabla 3

Rúbrica socioformativa para evaluar la pronunciación y fluidez (Fragmento)

\begin{tabular}{|c|c|c|c|c|c|}
\hline $\begin{array}{c}\text { Preguntas } \\
\text { (indicadores) }\end{array}$ & Preformal & Receptivo & Resolutivo & Autónomo & Estratégico \\
\hline $\begin{array}{l}\text { 1. Pronunciación } \\
\text { y fluidez }\end{array}$ & $\begin{array}{l}\text { Sólo hace uso } \\
\text { de oraciones } \\
\text { simples, muy } \\
\text { breves, } \\
\text { aisladas y } \\
\text { preparadas pre- } \\
\text { viamente, } \\
\text { utilizando } \\
\text { muchas pausas } \\
\text { para buscar } \\
\text { expresiones, } \\
\text { articular pala- } \\
\text { bras y corregir }\end{array}$ & $\begin{array}{l}\text { Usa oraciones } \\
\text { simples y muy } \\
\text { breves. Son } \\
\text { constantes las } \\
\text { pausas. } \\
\text { Su } \\
\text { pronunciación } \\
\text { y articulación } \\
\text { son, } \\
\text { generalmente, } \\
\text { bastante claras } \\
\text { y } \\
\text { comprensibles, } \\
\text { aunque su }\end{array}$ & $\begin{array}{l}\text { Constantes } \\
\text { pausas, ya que } \\
\text { tiene algunos } \\
\text { problemas } \\
\text { para formular } \\
\text { su discurso, } \\
\text { pero puede } \\
\text { seguir } \\
\text { adelante. } \\
\text { La } \\
\text { pronunciación } \\
\text { es clara, } \\
\text { aunque su } \\
\text { acento }\end{array}$ & $\begin{array}{l}\text { Se expresa } \\
\text { con relativa } \\
\text { facilidad, } \\
\text { aunque tiene } \\
\text { dudas mientras } \\
\text { busca estruc- } \\
\text { turas o } \\
\text { expresiones. } \\
\text { La } \\
\text { pronunciación } \\
\text { es clara, } \\
\text { aunque su } \\
\text { acento } \\
\text { extranjero es }\end{array}$ & $\begin{array}{l}\text { Se comunica } \\
\text { con relativa } \\
\text { fluidez y, } \\
\text { aunque haga } \\
\text { pausas, es } \\
\text { capaz de } \\
\text { mantener el } \\
\text { ritmo } \\
\text { eficazmente. } \\
\text { Su } \\
\text { pronunciación } \\
\text { es clara, } \\
\text { aunque su } \\
\text { acento }\end{array}$ \\
\hline
\end{tabular}




\begin{tabular}{|c|c|c|c|c|}
\hline $\begin{array}{l}\text { la } \\
\text { comunicación. } \\
\text { Su } \\
\text { pronunciación } \\
\text { y articulación } \\
\text { solo son } \\
\text { correctas en } \\
\text { palabras y } \\
\text { frases me- } \\
\text { morizadas. Es } \\
\text { necesario hacer } \\
\text { un esfuerzo } \\
\text { para }\end{array}$ & $\begin{array}{l}\text { acento y sus } \\
\text { errores } \\
\text { ocasionales } \\
\text { pueden hacer } \\
\text { que su } \\
\text { interlocutor se } \\
\text { esfuerce por } \\
\text { comprenderle }\end{array}$ & $\begin{array}{l}\text { extranjero es } \\
\text { evidente, pero } \\
\text { comete } \\
\text { bastantes } \\
\text { errores. }\end{array}$ & $\begin{array}{l}\text { evidente y } \\
\text { puede } \\
\text { presentar } \\
\text { problemas con } \\
\text { algunos } \\
\text { sonidos. }\end{array}$ & $\begin{array}{l}\text { extranjero es } \\
\text { evidente y } \\
\text { comete muy } \\
\text { pocos errores }\end{array}$ \\
\hline
\end{tabular}

\section{Consideraciones éticas}

En la aplicación de los instrumentos se respetaron los lineamientos relacionados con el bienestar de los sujetos y todas disposiciones legales de protección de datos personales (American Psychological Association [APA], 2010).

\section{Resultados}

Los resultados se exponen en tres partes: 1) un análisis cuantitativo sobre el nivel de desarrollo de la competencia oral, 2) una descripción de los niveles predominantes según la rúbrica en todas los componentes, y 3) una explicación de las dificultades, explicadas por los docentes desde su experiencia como instructor.

\section{Análisis cuantitativos sobre el nivel de desarrollo de la competencia oral de la muestra estudiada}

Se presentan en la Tabla 4 los datos cuantitativos en la evaluación de cada componente y de cada nivel. Posteriormente se hace un análisis de los datos 


\section{Tabla 4}

Evaluación de la competencia oral

\begin{tabular}{|c|c|c|c|c|c|}
\hline Indicadores / Nivel & Preformal & Receptivo & Resolutivo & Autónomo & Estratégico \\
\hline Vocabulario (V) & $41 \%$ & $20 \%$ & $9 \%$ & $15 \%$ & $15 \%$ \\
\hline \multicolumn{6}{|c|}{ Competencia gramatical } \\
\hline (CG) & $28 \%$ & $25 \%$ & $21 \%$ & $19 \%$ & $07 \%$ \\
\hline \multicolumn{6}{|c|}{ Pronunciación y fluidez } \\
\hline$(\mathbf{P F})$ & $29 \%$ & $24 \%$ & $20 \%$ & $17 \%$ & $10 \%$ \\
\hline \multicolumn{6}{|c|}{ Coherencia y cohesión } \\
\hline (CC) & $29 \%$ & $24 \%$ & $20 \%$ & $13 \%$ & $14 \%$ \\
\hline Interacción (IN) & $21 \%$ & $20 \%$ & $19 \%$ & $28 \%$ & $12 \%$ \\
\hline
\end{tabular}

Elaboración propia

Los resultados muestran que los estudiantes tienen un nivel bajo en todas las dimensiones. En todas ellas, poco más de la mitad de los evaluados están en los niveles bajos (preformal y receptivo), mientras que solo en IN (interacción), menos de la mitad se encuentran en dichos niveles bajos (41\%). También se puede notar que, en todas las dimensiones, a excepción de IN, el grupo que contiene el porcentaje más alto de alumnos es el del nivel preformal y en todos, ese grupo es poco menor a un tercio de los evaluados. En la dimensión V (vocabulario), en cambio, el porcentaje es mayor a la tercera parte, en donde 4 de cada 10 alumnos está en el nivel más bajo. Analizando la tabla se observa que el vocabulario es donde los evaluados tuvieron más dificultades con un $41 \%$ seguido de la pronunciación y fluidez y de la coherencia y cohesión con $29 \%$ cada uno después viene la gramática con un $28 \%$ seguido de la interacción con un $21 \%$.

Las carencias de vocabulario generan también dificultades en cuanto a fluidez y pronunciación. Por una parte, el escaso acervo léxico impide que el discurso de desenvuelva de forma ligera y continua; por otra, el insuficiente dominio de los términos franceses retrasa la apropiación de las peculiaridades fonéticas que difieren notablemente de la lengua materna, como la articulación de las vocales y los sonidos nasales o guturales. Esa situación se observa, en general, en los principiantes, sin embargo, se pudo constatar que prevalece aun en algunos estudiantes que aspiran al nivel B1. 
En contraparte se pudo constatar que, aunque el vocabulario es el punto más deficiente, en general, también es el aspecto en el que hay el más alto porcentaje en el nivel estratégico (15\%). Eso comprueba que el vocabulario es un aspecto clave en el desarrollo de la habilidad lingüística, del que depende el desarrollo de los otros aspectos. Después del vocabulario sigue la (CC) coherencia y cohesión con $14 \%$ y la interacción con $12 \%$. La interacción obtuvo en general un porcentaje elevado en los niveles superiores de la rúbrica. Eso se puede explicar por el hecho de que las características principales de la interacción que son las normas y prácticas sociales, como el respecto al turno de hablar, la colaboración, el saber escuchar y responder al interlocutor de forma apropiada son de carácter universal e independientes del empleo de la lengua hablada, por lo que era de esperarse que los resultados revelaran niveles relativamente altos de dominio.

\section{Descripción de las dificultades predominantes según la rúbrica en todas los componentes}

De acuerdo con los descriptores de la rúbrica, las dificultades se pueden caracterizar por:

-un vocabulario limitado y repetitivo o con el uso de palabras poco precisas, lo que hace difícil la transmisión de la información requerida;

- confusiones o mal uso de palabras por interferencia de la lengua materna, como télévisive (de télévision), se matriculer (s'inscrire), elle a fait 50 ans (elle a eu); -algunos empleos inapropiados sobre el género y los posesivos, también ligados a las interferencias con la lengua materna, por ejemplo, el uso de "le" al lugar de "la" en ciertas palabras como "le voiture" que en francés es un sustantivo femenino, - incorrecciones en la utilización de las preposiciones de los países como "en France”, "à París" "au Mali”. Los evaluados tuvieron la tendencia de utilizar la preposición "en" para todos, es decir, para los países masculinos, femeninos y para las ciudades; - confusiones en la elección correcta entre los dos verbos auxiliares para la formación del pasado compuesto.

- omisión de los artículos partitivos " $d u$, de la, des, de l ” como, por ejemplo, "je mange poulet" al lugar de "je mange du poulet", o de la partícula "pas" en la negación como 
"je ne suis ètudiant" al lugar de "je ne sui spas ètudiant", probablemente por ser formas inexistentes en el castellano.

- dificultades en la sintaxis de ciertas frases como "un très important examen" (un examen très important), “ils se vont marier" (ils vont se marier).

-dificultades con las palabras terminadas en "tion" en general como "èducation", "tradition";

- una pronunciación de las consonantes finales de la " $e$ ";

- falta de la vibración de las cuerdas vocales que da lugar a confusiones entre el sonido " $s$ " que es una consonante sorda y " $z$ " que es una consonante sonora como "dessert"

y "desert" que son dos palabras totalmente diferentes, uno significa el desierto y el otro significa el postre;

- problemas de organización de las ideas por falta de conectores para expresar sus puntos de vista o impresiones con claridad;

- problemas de estructura del discurso, es decir una introducción, un desarrollo y una conclusión. Estas últimas dificultades reflejan carencias en el aspecto coherencia y cohesión.

\section{Explicación de las dificultades}

Los datos recopilados en las entrevistas con los evaluadores revelaron que existen 3 principales explicaciones de las dificultades que los alumnos hispanoparlantes enfrentan para el manejo de la competencia oral: 1) recurso al vocabulario de la lengua materna, 2) interferencia del inglés que es enseñado en México como lengua extranjera, 3) El temor de tener un docente nativo. Acerca de las dificultades, los evaluadores comentaron lo siguiente:

\section{1). Recurso al vocabulario de la lengua materna}

Los estudiantes ya tienen una lengua que les sirve de referencia para adquirir la segunda lengua, ya tienen un sistema fonético y fonológico, un sistema de escritura, unas categorías lingüísticas y otras normas y así el aprendizaje de la segunda lengua se ve mediado por el conocimiento lingüístico de esa primera y por lo tanto los estudiantes aprovechan este conocimiento de base para construir la nueva. Existe, además cierta proximidad entre las palabras del francés y el español, por ejemplo, Accesorio - accessoire: en el momento de procesar la información de la palabra el cerebro analiza a primera vista algo similar al español 
cuya diferencia significativa reside en la -e final que los estudiantes asimilaron. Así lo expresaron los evaluadores:

[E1]: Se observa que cuando mencionan una frase y hay una palabra que no la saben lo dicen en su lengua natal o en inglés.

[E8]: Hay muchas palabras poco presentes en los manuales y unidades pero que al final los estudiantes intentan de usar, van a lo más sencillo y lo afrancesan.

\section{2). Interferencia del inglés que es enseñado en México como lengua extranjera.}

Este segundo factor es muy relacionado con el primero. Existe el problema de transferencia negativa o interferencia que lleva a que los estudiantes que pretenden expresarse con fluidez prefieren utilizar los términos o estructuras que son familiares para ellos. Este factor se manifiesta en general con errores de omisión, problemas de estructuras, o errores de adición.

[E16]: Los estudiantes ven los idiomas extranjeros pensando solamente en la lengua inglesa que es en general la primera lengua extranjera que aprenden. Esto conlleva que sigan la sintaxis inglesa más que la francesa.

\section{3). El temor de tener un docente nativo}

Contrariamente a lo que se piensa que los estudiantes prefieren estar en contacto con maestros nativos, varios estudiantes manifestaron tener miedo de expresarse cuando tienen un instructor nativo ya que manifiestan que ellos tienen poca tolerancia a los errores y la comunicación ante las dudas resulta más fácil con un docente no nativo.

[E15]: Los estudiantes señalan que los nativos hablan demasiado rápido y son menos tolerante con los errores sobre todo en la pronunciación por eso prefieren un maestro no nativo a los niveles básicos.

\section{Conclusión}

La rúbrica que se utilizó es un instrumento que permite recoger simultáneamente datos cuantitativos y cualitativos, lo cual favorece un análisis más completo para identificar las dificultades y contar con elementos desde los que se puedan generar estrategias de transformación y mejora de los procesos formativos para el desarrollo de las habilidades comunicativas de los estudiantes de francés en contextos hispanoparlantes, específicamente las relacionadas con la competencia oral. 
El estudio mostró que la dificultad mayor es la referente al vocabulario, y eso resulta bastante lógico, puesto que, sin un buen repertorio lexical no es posible la construcción correcta de estructuras gramaticales ni la fluidez en la comunicación. También reveló dos importantes factores que influyentes en esas dificultades, según la opinión de los docentes evaluadores, que son la influencia tanto de la lengua materna como del aprendizaje del inglés como segunda lengua. Esos resultados coinciden con una parte de la conclusión de los estudios de Al Mustafá (2016) que señaló dos factores que son el miedo de hablar y la influencia de la lengua materna como fuentes de las dificultades. También se pueden comparar con los resultados de Asma (2016) quien señaló la falta de vocabulario como principal dificultad.

Es importante señalar que una limitación de este estudio radica en que la muestra y los entrevistados pertenecen a una misma unidad de la $\mathrm{AF}$, lo que hace difícil encontrar relaciones y generalizaciones significativas a partir de los datos. Además, la evaluación se limitó a los aspectos del desempeño en la competencia oral, situación que complica determinar el nivel general en otros componentes del dominio de la lengua francesa en los evaluados.

A pesar de que el presente estudio representa una aportación importante para identificar las dificultades de la competencia oral es muy necesario promover nuevos estudios ampliando la muestra a estudiantes de otras unidades de la AF en México o en el mundo y complementar la evaluación de diagnóstico obteniendo otros datos de los alumnos, como antecedentes académicos, antecedentes lingüísticos, estancias internacionales previas, empleo del tiempo libre, oportunidad de exposición o práctica de la lengua en otros espacios. También se recomienda realizar nuevos estudios específicos sobre cada una de las dificultades mencionadas.

\section{Referencias}

Al Hosni, S. (2014). Speaking Difficulties Encountered by Young EFL Learners. International Journal on Studies in English Language and Literature, 2(6), 22-30. Recuperado de: https://urlz.fr/9WVh

Al Mustafá, M (2016). Difficultés de l'Expression Orale dans la classe du (FLE). (Mémoire de master, Université du Soudan de Sciences et de Technologie, Omdurman Ahlia, Soudan. Recuperado de : https://urlz.fr/aftB

American Psychological Association. (2010). Manual de publicaciones de la American Psychological Association. (6a ed.). México: Manual Moderno. 
Asma, M. (2016). Les difficultés de la production orale Chez les apprenants de la 2ème année moyenne. (Mémoire de master, Université Mohamed Khider, Biskra, Algeria). Recuperado de : https://urlz.fr/ajKy

Bygates, M. (1991). Speaking. Reino Unido: Oxford University Press.

Consejo de Europa. (2002). Marco Común Europeo de Referencia para las Lenguas:

Aprendizaje, enseñanza, evaluación. Madrid: Instituto Cervantes. Recuperado de: https://urlz.fr/9TOq

Hrisa, M., \& Pop-Pacurar, I (2016). Is the fear of "BeingWrong" a Barrier for Effective Communication between Students and Professors? A Survey Study at Babes-Bolyali University Romania. Acta Didactica Napocensia, 9(2), 47-66. Recuperado de:https://urlz.fr/9WS4

Mejía, D. (2014). Factores de dificultad para el aprendizaje del inglés como lengua extranjera en estudiantes con bajo rendimiento en inglés de la Universidad Icesi" (proyecto de grado de maestría en educación) Universidad ICESI, SANTIAGO DE CALI

Mounkoro, I. \& Herrera-Meza, S. (2019). Validez y Confiabilidad de una Rúbrica Socioformativa para Evaluar la Competencia Oral en Segunda Lengua. (en prensa).

Níkleva, D. G., \& López-García, M. P. (2019). El reto de la expresión oral en Educación Primaria: características, dificultades y vías de mejora. Educatio Siglo XXI, 37(3 Nov- Feb), 9-32. https://doi.org/10.6018/educatio.399141

Níkleva, D. G., \& López-García, M. P. (2019). El reto de la expresión oral en Educación Primaria: características, dificultades y vías de mejora. Educatio Siglo XXI, 37(3 Nov- Feb), 9-32. https://doi.org/10.6018/educatio.399141

O'malley, J. y Valdez, P. (1996). Authentic assessment for English language Learners. United States: Addison-Wesley Pub. Co

Oxford, R. (1990). Language Learning Strategies: What Every Teacher Should Know. Boston, MA: Heinle \& Heinle.

Ramírez, J (2002). La expresión oral. Contextos educativos, 5(2002), 57-72. Recuperado de: https://urlz.fr/a27e

Sánchez, A. (2016) Obstáculos en el aprendizaje del inglés como lengua extranjera en dos grupos de población bogotana. (Trabajo de grado en la modalidad de monografía para optar al título de Licenciada en Lenguas Modernas Español-Inglés) Universidad distrital Francisco José de Caldas, BOGOTANA.

Scrivener, J. (1994). Learning teaching. Oxford: Macmillan Heinemann English Language Teaching. 
Tobón, S. (2013). Formación integral y competencias. Pensamiento complejo, currículo, didáctica y evaluación. 4ta. Ed. Bogotá: ECOE.

Tobón, S. (2015). La socioformación: Un estudio conceptual. México: CIFE.

Tobón, S. (2017). Evaluación socioformativa. Estrategias e instrumentos. Mount Dora (USA): Kresearch.

Tobón, S., Guzmán, C. E., Hernández, J. S., \& Cardona, S. (2015). Sociedad del conocimiento: Estudio documental desde una perspectiva humanista y compleja. Paradigma, 36(2), 7-36. Recuperado de: http://revistas.upel.edu.ve/index.php/paradigma/article/view/3023

Ur, P. (1996). A course in language teaching. Cambridge: Cambridge University Press.

Valero Molina, N. y Jiménez-Fernández, G. (2015). Estudio exploratorio sobre dificultades en el aprendizaje de una segunda lengua: la opinión del profesorado. Espiral. Cuadernos del Profesorado, 8(16), 3-12. Disponible en: http://www.cepcuevasolula.es/espiral.

Youcef, A. (2018). Les origines des difficultés à l'oral chez les étudiants de FLE (Mémoire présenté en vue de l'obtention du diplôme de Master en Didactique et langues appliquées) Université 8 Mai 1945 Guelma. République Algérienne Démocratique,et Populaire.

Zabalza, M. (2017) Evaluación del aprendizaje y papel del mediador. Conferencia presentada en el Congreso Internacional de Evaluación del Desempeño. Valora 2017. CIFE 